\title{
Electrically and thermally conductive thin elastic polymer foils containing SiC nanofibers
}

Sandra Paszkiewicz ${ }^{*}$, Iman Taraghi ${ }^{1,2}$, Anna Szymczyk ${ }^{3}$, Andrzej Huczko ${ }^{4}$, Magdalena Kurcz $^{4}$, Bartlomiej Przybyszewski ${ }^{5}$, Rafal Stanik $^{6}$, Amelia Linares $^{7}$, Tiberio A. Ezquerra ${ }^{7}$, Zbigniew Rosłaniec ${ }^{1}$

${ }^{1}$ West Pomeranian University of Technology, Institute of Material Science and Engineering, Piastow Av. 19, PL-70310 Szczecin, Poland

${ }^{2}$ Semnan University, Department of Mechanical Engineering, Semnan, 35131-19111, Iran

${ }^{3}$ West Pomeranian University of Technology, Institute of Physic, Piastow Av. 48, PL-70311 Szczecin, Poland

${ }^{4}$ Warsaw University, Department of Chemistry, Laboratory of Nanomaterials Physics and Chemistry, 1 Pasteur str., PL-02093 Warsaw, Poland

${ }^{5}$ Warsaw University of Technology, Faculty of Materials Science and Engineering, Woloska 141 Street, 02-507 Warsaw, Poland

${ }^{6}$ Technische Universität Dresden, Institute of Lightweight Engineering and Polymer Technology, Holbeinstraße 3, 01307 Dresden, Germany

${ }^{7}$ Instituto de Estructura de la Materia, IEM-CSIC, Serrano 121, 28006 Madrid, Spain

*Corresponding author. Tel.:+48 9144945 89; fax: +48 9144943 56, e-mail address:

spaszkiewicz@zut.edu.pl

Abstract: Design and experiment of polymeric nanocomposites (NCs) for photovoltaic applications with outstanding electrical and thermal properties has been investigated with the introduction of $\mathrm{SiC}$ nanofibers (NFs) into the poly(trimethylene terephthalate)-blockpoly(tetramethylene oxide) (PTT-PTMO) copolymers. In order to enhance the electrical and thermal conductivity, different concentrations of SiC NFs, ranging from 0.1 to $3.0 \mathrm{wt} \%$, have 
been selected to mix with PTT-PTMO via in situ polymerization method. This reaction method is an excellent choice for incorporation of high amount of SiC NFs (3 wt \%) into the polymer that was confirmed by morphological studies. From dielectric spectroscopy studies a percolating behavior was confirmed at low percolation threshold (less than $2 \%$ wt $\%$ ). Furthermore, the $15 \%$ increment for thermal conductivity appeared with combination of 0.5 wt \% SiC NFs with PTT-PTMO copolymers, which can be affected by manufacturing process of NCs, state of nanofillers dispersion and aspect ratio of nanofillers.

Keywords: thermoplastic elastomers; silicon carbide; electrical conductivity; thermal conductivity.

\section{Introduction}

Among all of the renewable energy sources solar photovoltaic technologies are the most promising. The strong demand for solar photovoltaic technologies is due to their abundant, inexhaustible, and clean characteristics, and possibility of usage in a great number of applications [1, 2]. Polymeric based solar cells have recently attracted much attention of scientists and industry on account of their several advantages such as scalability, flexibility, printability, and low cost [3-5]. Thin polymer foils have been used as transparent [6], semitransparent [7] and non-transparent [8] solar photovoltaic systems, in which the incorporation of nanomaterials can be a key factor to optimize the operation of solar cells $[9,10]$. Seo et al. [11] studied the relative improvement in power conversion efficiency of polymer nanocomposites (NCs) photovoltaic cells consisting of poly(3-hexylthiophene) (P3HT) and functionalized CdSe nanocrystals. Rath and Trimmel [12] carried out the in situ synthesis of semiconducting nanoparticles (NPs) in conjugated polymer matrices for photovoltaic applications, in which the hybrid solar cells based on conjugated polymers and inorganic semiconducting NPs combined beneficial properties of organic and inorganic semiconductors. Zhao et al. [13] prepared the hybrid polymer electrolyte nanocomposite with $\mathrm{SiO}_{2}$ nanofiber 
as the filler for solid state dye-synthesized solar cell. Moreover, polysiloxane/graphite NPs films, which thickness corresponds to the lateral dimension of the modified graphite NPs, were fabricated by Cho et al. [14], in which the controlled assembly of graphite nanosheets (GNs) (5 wt $\%$ ) enhanced the thermal diffusivity of the NCs by $330 \%$ and $200 \%$ compared to the pristine polymer and the corresponding NCs with a random distribution of GNs, respectively. On the other hand, Chen et al. [15] synthesized ultraviolet curable organic/inorganic hybrid NCs with good thermal stability, moderate adhesion strength, and excellent barrier capability for encapsulation of organic solar cells, using a capillary-driven particle-level templating technique, Heeder et al. [16] fabricated multi-functional polystyrene/graphite nanoplatelets NCs, in this case the results showed that this novel fabrication technique could produce a NC that possesses both excellent transport properties and improved mechanical strength. All these mechanical, thermal and electrical evaluations are made to estimate the critical conditions of the polymeric NCs constructions into the photovoltaic solar devices. Therefore, the challenge is to select the appropriate nanoparticles in the aforementioned systems to obtain the best possible efficiency of the process. Ceramic silicon carbide $(\mathrm{SiC})$ nanofibers, which improve electron mobility, thermal conductivity and further chemical and high temperature resistivity [17-19], can be an option for incorporation into novel polymeric materials to be used in photovoltaic utilizations. Moreover, $\mathrm{SiC}$ thin films are used in a wide range of applications such as high-power electronics and photovoltaic and optoelectronic devices [20]. Recently developed poly(trimethylene terephthalate)-blockpoly(tetramethyleneoxide) copolymers (PTT-PTMO), first synthesized and characterized by Szymczyk et al. [21], are materials that exhibit low glass transition temperature combined with high melting temperature of PTT crystallites even at high flexible segments contents, that make them suitable for low and high temperature range applications. When it comes to the research on the effects of different types of NPs on PTT-PTMO properties, among others 
Guskos et al. [22] investigated the temperature dependence of dc magnetization and ferromagnetic resonance of two samples containing c- $\mathrm{Fe}_{2} \mathrm{O}_{3}$ (maghemite) magnetic NPs dispersed at low concentration (0.1 and 0.3 wt \%) in PTT-PTMO matrix. Moreover, Paszkiewicz et al. [23-25] investigated the effect of three different types of 2D nanofillers i.e. montmorillonite (MMT), graphene nanoplatelets (GNP) and graphene oxide (GO) on structure and mechanical properties of NCs based on PTT-PTMO copolymer. In another work, the effect of polyhedral oligomeric silsesquioxanes (POSS) on the microstructure and phase separation of PTT-PTMO copolymers was observed [26], in which thermal analysis imply that POSS cages in PTT-PTMO copolymer act as anti-nucleation agents and inhibit the crystallization of PTT hard phase during the cooling down process from the melt shifting crystallization temperature toward lower values. Furthermore, the addition of single walled carbon nanotubes (SWCNT) and GNP enhanced the electrical conductivity of PTT-PTMO matrix, particularly in the case of NCs with 0.3 wt. \% SWCNT and 0.1 wt. \% GNP. Additionally, it was found that when the ratio of SWCNT to GNP was 5:1, i.e. $0.5 \mathrm{wt} \%$ of SWCNT and 0.1 wt \% of GNP, the PTT-PTMO-based NCs exhibited an increase of thermal conductivity of ca $30 \%$ if compared to neat PTT-PTMO [27]. This suggests that the combination of two types of nanofillers, which differ in shape, allows to obtain the synergistic effect for the electrical and thermal conductivity enhancement of PTT-PTMO copolymer.

The main objective of this study was to obtain electrically and thermally conductive elastic thin polymer foils that can be used in photovoltaic devices. The flexibility and high thermal resistance capability of the PTT-PTMO copolymers are the crucial features to make these materials suitable candidates for incorporation of NPs in photovoltaic devices. To the best knowledge of the authors, the study on the effect of SiC NPs on the electrical and thermal conductivity of PTT-PTMO copolymer is reported for the very first time and further 
discussions about the morphology and characteristics of the NCs are brought in the next sections.

\section{Experimental methods}

\subsection{Materials and synthesis procedure}

$\mathrm{SiC}$ nanofibres were produced via self-propagating high-temperature synthesis (SHS) from elemental Si and poly(tetrafluoroethylene) (PTFE) powder mixtures. The synthesis is highly exothermic redox reaction, where PTFE is an oxidant and Si is a reducer. The reaction follows a scheme presented below:

$$
2(\mathrm{CF})_{2}+2 \mathrm{Si} \rightarrow \mathrm{SiC}+\mathrm{SiF}_{4}+\mathrm{C}
$$

The presented scheme is a simplification since the reaction runs at both high temperatures and high pressures and, most likely, under non-equilibrium conditions. It is a multistage process and products are not limited to those above presented because some fluorides and soot can be also produced. However, SiC nanofibers are the main product. The combustions were carried out in a modified calorimeter bomb, a stainless steel reactor with volume of $2700 \mathrm{~cm}^{3}$. Detailed description of the reactor is presented elsewhere [28]. The reactants (PTFE and Si, 36\%:64\%) were well mixed, loaded into the quartz crucible and moved into the reactor, than the reactor was filled with combustion gas $-\mathrm{CO}$ at a pressure about $10 \mathrm{~atm}$ (the pressure which was previously defined as optimal [28]). The reaction was started by resistive heating of carbon thread. After the ignition the reaction ran in an exothermic mode. The whole synthesis lasted about few seconds. The averaged combustion temperatures were evaluated using

emission spectroscopy to be above $2000 \mathrm{~K}$. After the synthesis the product was collected and purified following a previously established protocol [29]. 
NCs based on PTT-PTMO block copolymer with SiC NFs were synthesised by melt transesterification and subsequently polycondensation following the procedure described previously [21, 23-26]. Before the synthesis, an appropriate amount of nanofibers was dispersed in bio-1,3-propanenediol (bio-PDO) (Susterra®Propanediol, DuPont Tate\&Lyle, USA) using ultra-high speed stirrer (Ultra-Turax T25) and sonicator (Homogenizer HD 2200, Sonoplus, with a frequency of $20 \mathrm{kHz}$ and $75 \%$ of power $200 \mathrm{~W}$ ) in each case for $15 \mathrm{~min}$. Then the first step, the transesterification reaction, was carried out, i.e. the prepared dispersion in bio-PDO, along with dimethyl terephthalate (DMT, Sigma-Aldrich) and tetrabutyl orthotitaniate (TBT, Fluka) catalyst was loaded into $1 \mathrm{dm}^{3}$ steel reactor (Autoclave Engineers Inc, USA). This stage was conducted under nitrogen flow in the temperatures range of 165$180{ }^{\circ} \mathrm{C}$ for 2.5 hour in the presence of catalyst. When the first step was completed, the poly(tetramethylene oxide) glycol with a molecular weight of $1000 \mathrm{~g} / \mathrm{mol}$ (PTMG, Terathane 1000, DuPont, USA), thermal stabilizer Irganox 1010 (Ciba-Geigy, Switzerland) and second portion of catalyst (TBT) were added. At this stage, the polycondensation reaction was being proceeded at a temperature of $250{ }^{\circ} \mathrm{C}$ under reduced pressure of $\sim 15 \mathrm{~Pa}$. Synthesis was finished when the melt reached a specified melt viscosity's value, which corresponds to the high molecular weight copolymer. The obtained $\mathrm{NC}$ was extruded from the reactor under nitrogen flow in the form a streak, cooled to room temperature in the water bath and then granulated. The neat PTT-PTMO copolymer, which was the reference sample, was synthesized following the same procedure.

\subsection{Methods of characterization}

\subsubsection{X-Ray Diffraction (XRD)}

The XRD analysis has/have been performed for the purified, powder samples. The purification procedure is presented elsewhere [29]. The products were measured at XRD Powder Diffractometer D8 Discover (WAXS) $\mathrm{Cu}$ K $\alpha$ radiation, $\lambda=1.54 \AA$. 


\subsubsection{Transmission electron microscopy (TEM)}

The samples for TEM analysis were shortly sonicated in ethanol, then the solution was deposited on the TEM grids. The analyses were performed using transmission electron microscope Zeiss Libra 120.

\subsubsection{Scanning electron microscopy (SEM)}

The structure of SiC nanofillers (before treatment) and NCs were observed by scanning electron microscopy (SEM, JEOL JSM 6100 SEM). The samples were cryofractured in liquid nitrogen and then vacuum coated with a thin gold film before being analyzed using SEM.

\subsubsection{Dielectric spectroscopy}

Circular gold electrodes ( $2 \mathrm{~cm}$ in diameter) were deposited onto the surfaces of the film samples by the sputtering technique. The electrical characterization of the NCs was conducted by means of a Novocontrol broadband dielectric spectrometer in the frequency range from $10^{-}$

${ }^{2} \mathrm{~Hz}$ to $10^{7} \mathrm{~Hz}$, at room temperature. The complex dielectric permittivity $\varepsilon^{*}=\varepsilon^{\prime}-i \varepsilon^{\prime \prime}$, where $\varepsilon^{\prime}$ represents the permittivity and $\varepsilon^{\prime \prime}$ the dielectric loss, was measured as a function of frequency $(\mathrm{F})$ of the applied electric field. Electrical conductivity was calculated from equation : $\sigma(F)=\varepsilon_{0} 2 \pi F \varepsilon^{\prime \prime}$, where $\varepsilon_{0}$ is the vacuum permittivity [30].

\subsubsection{Thermal conductivity measurements}

To determine the thermal conductivity coefficient of polymer materials, the transient plane source (TPS) method, the Hot Disk TPS 2500 S (Uppsala, Sweden), and the Hot Disk thermal constants analyzer were used according to ISO 22007-2. The details of the measurement are presented in [27]. The measurements were performed on the middle part of a dumb-bell shape sample (type A3) with the penetration depth of 3.5-3.9 $\mu \mathrm{m}$. All specimens with a thickness of approximately $2 \mathrm{~mm}$ were placed on both sides of the Hot Disk sensor touching the sensor with plane surfaces.

\section{Results and Discussion}




\subsection{Morphological characterization}

Fig. 1a shows the SEM image of SiC nanofibers before purification. The presented material was collected from the SiC rich area - whitish-greenish coating on the raw product. The XRD spectra (Fig. 1b), measured for purified product, confirmed the presence of SiC. The signals are characteristic for $\beta$ - $\mathrm{SiC}\left(2 \Theta=35.6^{\circ}\right.$ for $\mathrm{SiC}\left(\begin{array}{lll}1 & 1 & 1\end{array}\right), 41.4^{\circ}$ for $\mathrm{SiC}\left(\begin{array}{lll}2 & 0 & 0\end{array}\right)$ and $60.0^{\circ}$ for $\mathrm{SiC}$ (2 2 0)). Some residual, left after the purification process, co-product $\mathrm{C}$ and unreacted $\mathrm{Si}$ are also visible. While the XRD measurements confirmed a composition of the product, TEM (Figs. $1 \mathrm{c}-\mathrm{d}$ ) proved its morphology. 1D nanostructures' typical diameters are in the range of 10 to $100 \mathrm{~nm}$, while their lengths are up to several microns, so their aspect ratio is well above $10^{3}$. SiC exhibits unique physical properties such as thermal and chemical resistance, high mechanical and electrical performance, which make it a promising candidate for electronics, composites or other high-temperature and harsh-environment applications [31]. SiC nanofibers, being 1D nanostructures, possess new properties resulting from unique shape and nanoscale-quantum-confinement effects.

Moreover, the morphology evolution of the PTT-PTMO/SiC NCs filled with $0.5 \mathrm{wt} \%, 1$ wt $\%$ and 3 wt $\%$ is depicted in Figs. 2a-c, respectively. SiC NFs were distributed in the matrix, with no obvious agglomerations. The fracture surface of the specimens showed the randomly distributed $\mathrm{SiC}$ in the PTT-PTMO matrix, in which the size of NFs was about several microns. Regardless of the concentration of SiC NFs a good degree of their distribution in the polymer matrix was obtained. As similar trend reported for the combination of SiC NFs in poly(caprolactone) was due to an increase in the amount of NFs placed into the free space of polymer matrix [32]. Moreover, most of the SiC NFs were embedded in the polymer matrix with both ends. Thus, the SEM image of NCs surface suggests the "pulling" of the SiC NFs from the polymer matrix during the brittle fracture of the sample [27]. In Fig. 2c, the SiC NFs appear fully connected to each other forming a continuous network. 


\subsection{Broadband electrical conductivity}

Fig. 3 represents (a) the broadband electrical conductivity $(\sigma(F))$ and $(b)$ the dielectric constant $\left(\varepsilon^{\prime}\right)$, at room temperature, as a function of frequency $(\mathrm{F})$ for nanocomposites with different weight concentration of $\mathrm{SiC}$ [33]. For the matrix PTT-PTMO, at low frequencies, a characteristic conducting behavior (a frequency independent component, $\sigma_{\mathrm{dc}}$ ) associated with the presence of PTMO has been observed. Such behavior may be due to the ionic conductivity of PTMO [34]. At higher frequencies, above $10 \mathrm{~Hz}, \sigma(\mathrm{F})$ follows almost linear dependence with frequency with a slope close to 1 , which is characteristic for insulating materials. For the nanocomposites, at low nanoadditive contents, $\sigma(\mathrm{F})$ follows the same behavior as the polymer matrix. However, for higher concentrations, $\sigma(\mathrm{F})$ becomes independent of frequency in a wide range. Above a characteristic frequency, $F_{c}$, the dynamic conductivity follows a power law. This type of behavior, which was observed in a wide series of disordered solids, was investigated by Jonscher [35]. Consequently, it was designated as the "Universal Dielectric Response" (UDR). It is commonly described by the following equation:

$$
\sigma(\mathrm{F})=\sigma_{d c}+\sigma_{a c}=\sigma_{d c}+A F^{S}
$$

where $\sigma_{\mathrm{dc}}$ is the frequency-independent direct-current conductivity and the exponent $S$ takes values $0<S<1$. Continuous lines in Fig. 3a correspond to the fittings of Eq. 1 to the experimental data. From these fits, the $\sigma_{\mathrm{dc}}$ values can be extracted. As shown in Fig. 3b, for nanocomposites with high SiC content, the dielectric constant has higher values than those of pristine matrix. This fact suggests that there are many connections between fibers, with small gaps of polymer between them, which promotes polarization phenomena. Fig. 4 shows $\sigma_{\mathrm{dc}}$ data as a function of filler concentration for the nanocomposites investigated. As shown, a characteristic percolating behavior is observed, i.e. for low additive concentrations, the conductivity remains close to the level of the insulating matrix. With an increasing filler content, at a critical concentration $\left(\phi_{c}\right)$ where it is assumed that nanofibers form a continuous 
network, the conductivity exhibits a sudden insulator-conductor transition. The dc conductivity above the critical concentration can be analyzed in terms of the percolation theory [36] by means:

$$
\sigma_{\mathrm{dc}} \propto\left(\varnothing-\emptyset_{\mathrm{c}}\right)^{\mathrm{t}}
$$

where $t$ is a critical exponent. Although the critical concentration $\left(\phi_{c}\right)$ depends on the lattice in which "particles" are located, the critical exponent "t" depends primarily on the dimensionality of the percolating system and not on the details of the geometric structures or the interactions. The dashed line in Fig. 4 is the fit of Eq. 2 to the experimental data with $t=$ 1.7 and $\phi_{\mathrm{c}}=1.2$. Theoretical calculations, supported by a great number of experimental observations propose values of $t$ between 1.6 and 2 for three-dimensional systems [37]. Electrical conductivities higher than that of the insulating matrix, before a continuous "particle" network is formed, could be possible provided that an interparticle conduction mechanism like for example tunneling is present. This is more likely the origin of the observed departure of the conductivity values, at low concentrations, from the expected ones according to the percolation model. A similar effect was previously reported for polyethylene composites based on MWCNT and CNF [38].

\subsection{Thermal Conductivity}

Fig. 5 represents the thermal conductivity and thermal diffusivity of PTT-PTMO copolymer as a function of $\mathrm{SiC}$ content. One can see that the introduction of SiC NFs in the PTT-PTMO copolymer increases sharply the thermal conductivity of the NCs when the amount of SiC increases from 0 to $0.5 \mathrm{wt} \%$. The $\mathrm{SiC}$ NFs can effectively be accommodated in the polymer phase to form thermal conductive paths [39]. Moreover, the SiC NFs which are embedded in the polymer matrix can act as the barrier to heat transmission. Therefore, there is a critical concentration for the $\mathrm{SiC}$ content up to which the thermal conductivity increased dramatically due to the formation of a continuous network across the sample. For higher concentrations the 
rate of increment will be slighter. This significant increase in thermal conductivity of PTTPTMO/SiC was probably achieved by several reasons. Firstly, the features of a crystalline structure (PTT), which is expected to strongly influence heat transfer in both the polymer phase at the interface between SiC NFs and polymer. Secondly, the homogeneous distribution of SiC NFs (confirmed by SEM observations) and obtainment of an interconnecting network suitable for heat transfer [27]. Thirdly, the method of distribution of SiC NFs throughout the polymer matrix: herein the in situ polymerization method has been used to obtain the optimum results for the thermal conductivity of the PTT-PTMO copolymers [40]. The thermal diffusivity corresponds to the speed of the heat transfer in the PTT-PTMO is improved with the increase in the concentration of SiC NFs into the polymer matrix. In other words, the capability of the NCs for the propagation speed of thermal diffusion is raised up to $42 \%$ with addition of $3 \mathrm{wt} \% \mathrm{SiC}$ NFs.

\section{Conclusions}

The study on the morphological of SiC NPs dispersion in the matrix revealed the high capability of the PTT-PTMO block copolymer to incorporate significant amount of SiC with a good state of dispersion. From dielectric spectroscopy measurement, it can be concluded that these nanocomposites exhibit a percolating behavior with a percolation threshold as low as $1.2 \mathrm{wt} \%$ of $\mathrm{SiC}$ NFs. Moreover, the fact that these nanocomposites present higher dc conductivity than PTT-PTMO, in a certain nanofiber concentration range below the percolation threshold, suggests that a tunneling mechanism for charge transport is present. The rather low percolation threshold, the low cost of preparation of SiC NFs compared to nanocomposites containing SWCNTs (ca. $1000 \mathrm{USD} / \mathrm{kg}$ for SiC NFs and about hundreds USD/g for SWCNT) [41] and their low viscosity values, which makes it easier for extrusion process after in situ polymerization [42], give rise to a new class of interesting materials with potential use in a wide range of applications. 


\section{Acknowledgments}

Sandra Paszkiewicz would like to thank for financial support from West Pomeranian University of Technology (Dean's Grant for Young Scientists). Amelia Linares and Tiberio A. Ezquerra acknowledge the financial support provided by MINECO (grant MAT201566443-C02-1-R). Authors would like to thank DuPont Tate \& Lyle BioProducts company for providing bio-1,3-propanediol for experimental use.

\section{References}

[1] B. Parida, S. Iniyan, R. Goic, A review of solar photovoltaic technologies, Renew Sustain Energy Rev. 15 (2011) 1625-1636.

[2] R. Baños, F. Manzano-Agugliaro, F.G. Montoya, C. Gil, A. Alcayde, J. Gómez, Optimization methods applied to renewable and sustainable energy: A review, Renew Sustain Energy Rev. 15 (2011) 1753-1766.

[3] U. Mehmood, A. Al-Ahmed, I.A. Hussein, Review on recent advances in polythiophene based photovoltaic devices, Renew Sustain Energy Rev. 57 (2016) 550-561.

[4] H. Spanggaard, F.C. Krebs, A brief history of the development of organic and polymeric photovoltaics, Sol Energ Mat Sol C. 83 (2004) 125-146.

[5] J.W. Jung, J.W. Jo, E.H. Jung, W.H. Jo, Recent progress in high efficiency polymer solar cells by rational design and energy level tuning of low bandgap copolymers with various electron-withdrawing units, Org. Electron. 31 (2016) 149-170.

[6] C.C. Chen, L. Dou, R. Zhu, C.H. Chung, T.B. Song, Y.B. Zheng, S. Hawks, G. Li, P.S. Weiss, Y. Yang, Visibly transparent polymer solar cells produced by solution processing, ACS Nano. 6 (2012) 7185-7190.

[7] Z. He, C. Zhong, S. Su, M. Xu, H. Wu, Y. Cao, Enhanced power-conversion efficiency in polymer solar cells using an inverted device structure, Nature Photon. 6 (2012) 591-595. 
[8] A.C. Bedeloglu, A. Demir, Y. Bozkurt, N.S. Sariciftci, Photovoltaic properties of polymer based organic solar cells adapted for non-transparent substrates, Renew. Energ. 35 (2010) 2301-2306.

[9] G.B. Smith, C.A. Deller, P.D. Swift, A. Gentle, P.D. Garrett, W.K. Fisher, Nanoparticledoped polymer foils for use in solar control glazing, J. Nanopart. Res. 4 (2002) 157-165. [10] R.V. Salvatierra , C.E. Cava , L.S. Roman, A.J.G. Zarbin, ITO-free and flexible organic photovoltaic device based on high transparent and conductive polyaniline/carbon nanotube thin films, Adv. Funct. Mater. 23 (2013) 1490-1499.

[11] J. Seo, W.J. Kim, S.J. Kim, K.S. Lee, A.N. Cartwright, P.N. Prasad, Polymer nanocomposite photovoltaics utilizing CdSe nanocrystals capped with a thermally cleavable solubilizing ligand, Appl. Phys. Lett. 94 (2009) 133302.

[12] T. Rath, G. Trimmel, In situ syntheses of semiconducting nanoparticles in conjugated polymer matrices and their application in photovoltaics. Hybrid. Mater. 1 (2013) 15-36.

[13] X.G. Zhao, E.M. Jin, J.Y. Park, H.B. Gu, Hybrid polymer electrolyte composite with $\mathrm{SiO}_{2}$ nanofiber filler for solid-state dye-sensitized solar cells, Compos. Sci. Technol. 103 (2014) 100-105.

[14] H.B. Cho, D.P. Huynh, T. Nakayama, J.H. Shin, H. Suematsu, K. Niihara, K. Sung, Y. Lee, S. Kim, Y. Song, Y.H. Choa, Polymer nanocomposite films with thicknesses $(\leq 30 \mu \mathrm{m})$ corresponding to the lateral dimension of graphite nanosheets as straight forward thermal conducting pathways, Compos. Sci. Technol. 127 (2016) 106-112.

[15] C.M. Chen, M.H. Chung, T.E. Hsieh, M.O. Liu, J.L. Lin, W.P. Chu, R.M. Tang, Y.S. Tsai, F.S. Juang, Synthesis, thermal characterization, and gas barrier properties of UV curable organic/inorganic hybrid nanocomposites with metal alloys and their application for encapsulation of organic solar cells, Compos. Sci. Technol. 68 (2008) 3041-3046. 
[16] N. Heeder, A. Yussuf, I. Chakraborty, M.P. Godfrin, R. Hurt, A. Tripathi, A. Bose, A. Shukla, Fixed-angle rotary shear as a new method for tailoring electro-mechanical properties of templated graphene-polymer composites, Compos. Sci. Technol. 100 (2014) 70-75.

[17] Z. Guo, T.Y. Kim, K. Lei, T. Pereira, J.G. Sugar, H.T. Hahn, Strengthening and thermal stabilization of polyurethane nanocomposites with silicon carbide nanoparticles by a surfaceinitiated-polymerization approach, Compos. Sci. Technol. 68 (2008) 164-170.

[18] P. Mavinakuli, S. Wei, Q. Wang, A.B. Karki, S. Dhage, Z. Wang, D.P. Young, Z. Guo, Polypyrrole/Silicon Carbide Nanocomposites with Tunable Electrical Conductivity, J. Phys. Chem. C. 114 (2010) 3874-3882.

[19] K. Kueseng, K.I. Jacob, Natural rubber nanocomposites with SiC nanoparticles and carbon nanotubes, Eur. Polym. J. 42 (2006) 220-227.

[20] A. Kassiba, M. Makowska-Janusik, J. Bouclé, J.F. Bardeau, A. Bulou, N. Herlin-Boime, Photoluminescence features on the Raman spectra of quasistoichiometric $\mathrm{SiC}$ nanoparticles: Experimental and numerical simulations, Phys. Rew. B. 66 (2002) 155317.

[21] A. Szymczyk, E. Senderek, J. Nastalczyk, Z. Rosłaniec, New multiblock poly(etherester)s based on poly(trimethylene terephthalate) as rigid segments, Eur. Polym. J. 44 (2008) 436-443.

[22] N. Guskos, M. Maryniak, J. Typek, A. Guskos, R. Szymczak, E. Senderek, Z. Roslaniec, D. Petridis, K. Aidinis, Influence of maghemite concentration on magnetic interactions in maghemite/PTT-block-PTMO nanocomposite, J. Non-Cryst Solids. 354 (2008) 4401-4406.

[23] S. Paszkiewicz, I. Pawelec, A. Szymczyk, Z. Rosłaniec, Thermoplastic elastomers containing 2D nanofillers: montmorillonite, graphene nanoplatelets and oxidized graphene platelets, Pol. J. Chem. Technol. 17 (2015) 74-81.

[24] S. Paszkiewicz, A. Szymczyk, Z. Špitalsky', J. Mosnáceek, K. Kwiatkowski, Z. Rosłaniec, Structure and properties of nanocomposites based on PTT-block-PTMO 
copolymer and graphene oxide prepared by in situ Polymerization, Eur. Polym. J. 50 (2014) $69-77$.

[25] S. Paszkiewicz, I. Pawelec, A. Szymczyk, Z. Špitalsky’, J. Mosnáĉek, A. Kochmańska, Z. Rosłaniec, Effect of exfoliated graphite nanoplatelets' size on the phase structure, electrical, and barrier properties of poly(trimethylene terephthalate)-based nanocomposites, Polym. Eng. Sci. 55 (2015) 2222-2230.

[26] S. Paszkiewicz, R. Pilawka, B. Dudziec, M. Dutkiewicz, B. Marciniec, Agnieszka Kochmańska, R. Jedrzejewski, Z. Rosłaniec, Morphology and phase separation in PTT-blockPTMO nanocomposites containing POSS particles, Eur. Polym. J. 70 (2015) 37-44.

[27] S. Paszkiewicz, A. Szymczyk, R. Pilawka, B. Przybyszewski, A. Czulak, Z. Rosłaniec, Improved thermal conductivity of poly(trimethylene terephthalate-block- poly(tetramethylene oxide) based nanocomposites containing hybrid single-walled carbon nanotubes/graphene nanoplatelets fillers, Adv. Polym. Tech. (2015) 21611.

[28] A. Huczko, M. Osica, A. Rutkowska, M. Bystrzejewski, H. Lange, S. Cudziło, A selfassembly SHS approach to form silicon carbide nanofibres, J. Phys. Condens. Matter. 19 (2007) 395022.

[29] M. Kurcz, A. Huczko, M. Soszyński, rozdział, Self-Propagating High-Temperature Synthesis (SHS): A Simple Route to Carbon-Related Nanomaterials, in: O. Fesenko, L. Yatsenko (Eds), Nanophysics, Nanophotonics, Surface Studies, and Applications, the series Springer Proceedings in Physics, 2016, pp 559-578.

[30] F. Kremer, A. Schonhals, Broadband Dielectric Spectroscopy, Springer-Verlag, Heidelberg, 2003.

[31] M. Kurcz, A. Huczko, Węglik krzemu wczoraj, dziś, jutro, Przemysł Chemiczny, 6, 2012. 
[32] T.S. Mdletshe, S.B. Mishra, A.K. Mishra, Studies on the effect of silicon carbide nanoparticles on the thermal, mechanical, and biodegradation properties of poly(caprolactone), J. Appl. Polym. Sci. 132 (2015) 42145.

[33] J.K.W. Sandler, J.E. Kirk, I.A. Kinloch, M.S.P. Shaffer, A.H. Windle, Ultra-low electrical percolation threshold in carbon -nanotubes epoxy composites, Polymer 44 (2003) $5893-5899$

[34] S. Paszkiewicz, A. Szymczyk, X.M. Sui, H.D. Wagner, A. Linares, T.A. Ezquerra, Z. Rosłaniec, Synergetic effect of single-walled carbon nanotubes (SWCNT) and graphene nanoplatelets (GNP) in electrically conductive PTT-block-PTMO hybrid nanocomposites prepared by in situ polymerization, Compos. Sci. Technol. 118 (2015) 72-77.

[35] A.K. Jonscher, The Universal dielectric response. Nature. 267 (1997) 673-679.

[36] D. Stauffer, Scaling theory of percolation clusters, Phys. Rep-Rev. Sect. Phys. Lett. 54 (1979) 1-74.

[37] D. Stauffer, A. Aharony, Introduction to percolation theory, Taylor and Francis, London, 1992.

[38] A. Linares, J.C. Canalda, M.E. Cagiao, M.C. Garcia-Gutierrez , A. Nogales, I. MartinGullom, J. Vera, T.A. Ezquerra, Broad-band electrical conductivity of high density polyethylene nanocomposites with carbon nanoadditives: multiwall carbon nanotubes and carbon nanofibers, Macromolecules. 41 (2008) 7090-7097.

[39] J.P. Cao, X. Zhao, J. Zhao, J.W. Zha, G.H. Hu, Z.M. Dang, Improved thermal conductivity and flame retardancy in polystyrene/poly(vinylidene fluoride) blends by controlling selective localization and surface modification of SiC nanoparticles, ACS Appl. Mater. Interfaces. 5 (2013) 6915-6924. 
[40] N. Burger, A. Laachachi, M. Ferriol, M. Lutz, V. Toniazzo, D. Ruch, Review of thermal conductivity in composites: Mechanisms, parameters and theory, Prog. Polym. Sci. 61 (2016) $1-28$.

[41] H. Kim, A.A. Abdala, C.W. Macosko, Graphene/polymer nanocomposites, Macromolecules. 43 (2010) 6515-6530.

[42] A. Szymczyk , S. Paszkiewicz, Z. Rosłaniec, Influence of intercalated organoclay on the phase structure and physical properties of PTT-PTMO block copolymers, Polym. Bull. 70 (2013) 1575-1590. 


\section{LIST OF FIGURES}

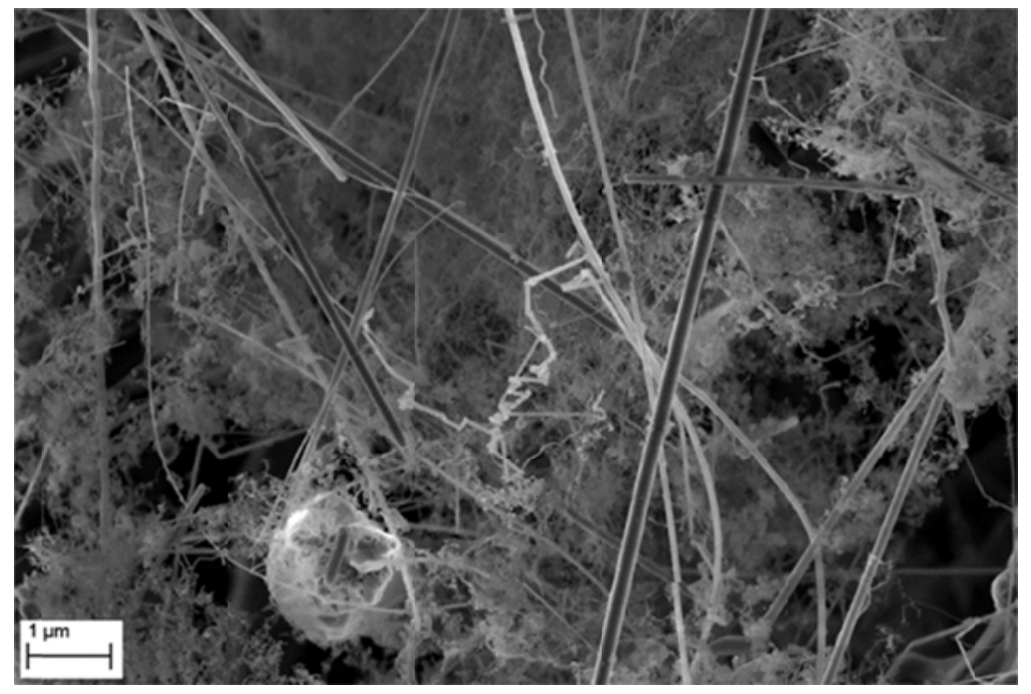

Fig. 1. (a) SEM image of as prepared SiC nanofibers (before purification) (scale bar is 200 $\mathrm{nm}$ ) and (b) XRD, (c) TEM (scale bar is $200 \mathrm{~nm}$ ) and (d) TEM (scale bar is $50 \mathrm{~nm}$ ) images of SiC nanofibers, after chemical purifying.
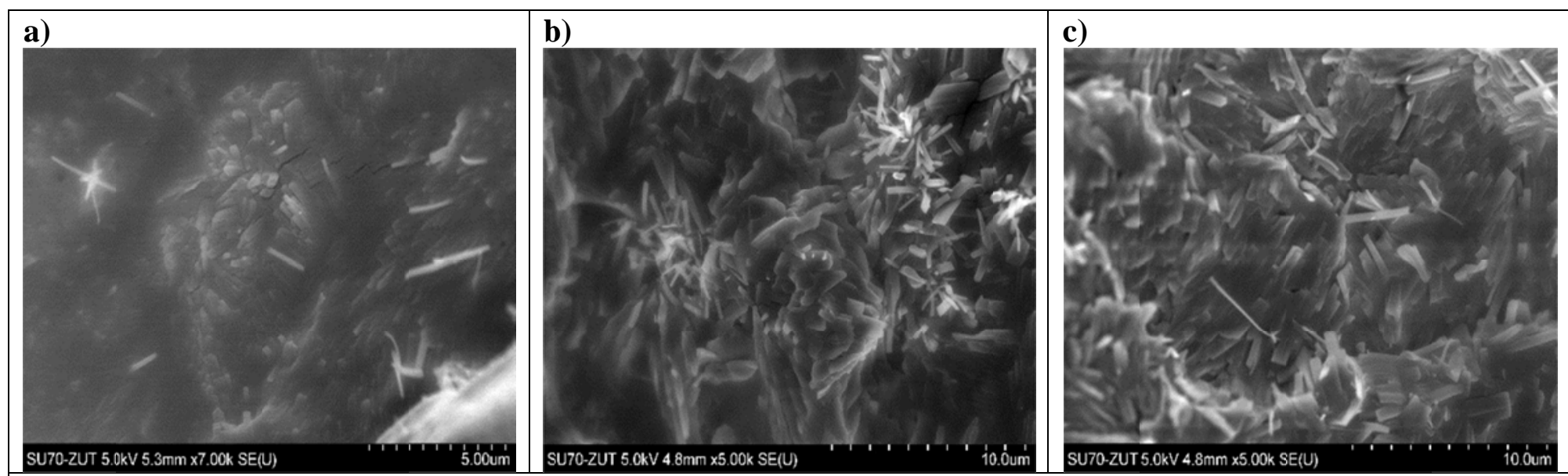

Fig. 2 SEM images of PTT-PTMO/SiC NCs reinforced with various weight percent of SiC NPs a) 0.5 wt \% b) 1.0 wt $\%$ c) 3 wt $\%$. 


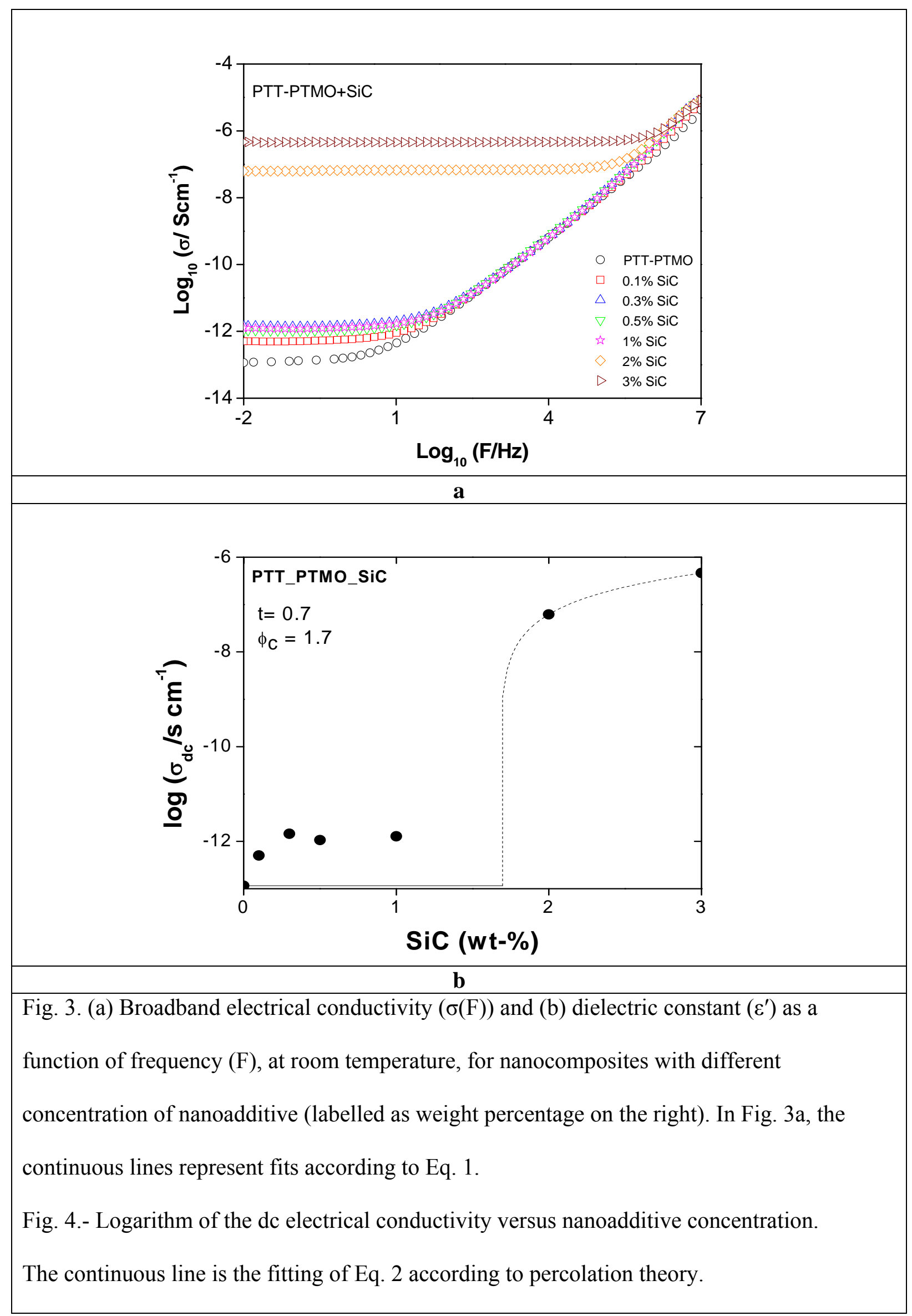




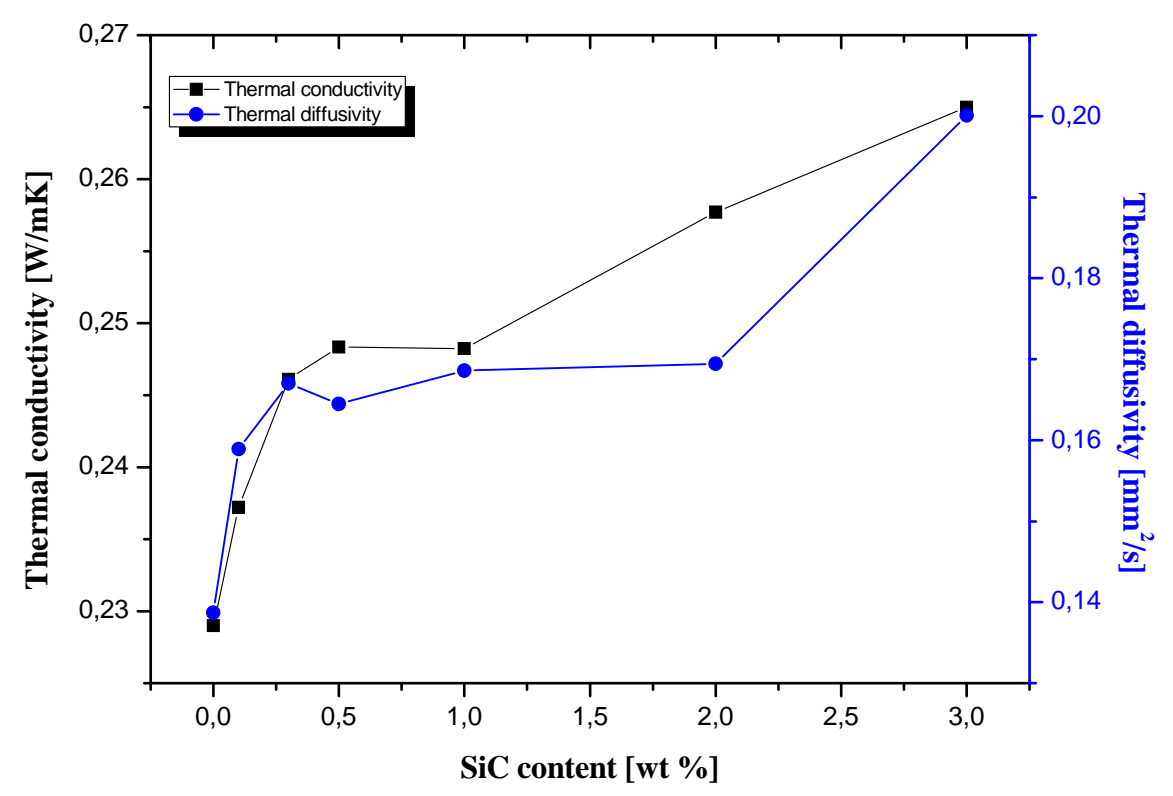

Fig. 5. Thermal conductivity and thermal diffusivity of PTT-PTMO block copolymer as a function of $\mathrm{SiC}$ content. 